\title{
The Quest for Usable Usability Heuristics for Game Developers
}

\author{
Sami Mylly, Mikko Rajanen and Netta Iivari
}

\begin{abstract}
Human Computer Interaction (HCI) research has provided many processes and methods for ensuring good usability during software development. The importance of usability in games has been acknowledged. However, there is a lack of research examining usability activities during actual game development and the suitability of different usability methods for different phases of game development. Game development industry, although growing fast and already including certain large and successful companies, consists of a huge number of small-to-medium-sized enterprises and start-ups. For these companies, practical, developer-oriented tools for ensuring game usability are needed, as these companies do not have resources for hiring usability specialists for taking care of usability. This paper reviews the concept of game usability and existing research on usability methods for game development. In addition, the paper proposes a set of usable usability heuristics as a practical, developer-oriented tool to be used during the game development process as well as reports on a small-scale survey study on game usability, heuristic evaluation and usability of usability heuristics in game development.
\end{abstract}

Keywords: Games · Usability · Heuristic Evaluation · Game Development · Game Design

Citation: Mylly S., Rajanen M., Iivari N. (2020) The Quest for Usable Usability Heuristics for Game Developers. In: Siarheyeva A., Barry C., Lang M., Linger H., Schneider C. (eds) Advances in Information Systems Development. ISD 2019. Lecture Notes in Information Systems and Organisation, vol 39. Springer, Cham. https://doi.org/10.1007/978-3-030-49644-9_10

A prior version of this paper has been published in the ISD2019 Proceedings (http://aisel.aisnet.org/isd2014/proceedings2019).

Sami Mylly

University of Oulu, Oulu, Finland

e-mail: noxious_productions@hotmail.com

Mikko Rajanen

University of Oulu, Oulu, Finland

e-mail: mikko.rajanen@oulu.fi

Netta Iivari

University of Oulu, Oulu, Finland

e-mail: netta.iivari@oulu.fi

(C) Springer International Publishing Switzerland 2019

A. Siarheyeva et al. (eds.), Advances in Information Systems Development - Information Systems Beyond 2020

DOI: https://doi.org/Xx.Xxxx/Xxx-X-Xxx-Xxxxx-X_xx 


\section{Introduction}

Computer and video games have extensively entered our everyday life. Game development industry is growing rapidly and has become very competitive. During the last twenty years games and their development have changed a lot in scope and in requirements [5]. Game development ranges from an enthusiastic individual creating a simple game as a spare time hobby to large software projects costing hundreds of millions and having hundreds of personnel. The estimated global sales of the game industry has surpassed 100 billion US dollars [49]. Game industry has also grown rapidly. For example, in Finland there were 150 game companies in 2012, 40\% of those started within the last two years [25], which has grown to 250 active studios in 2016 [26]. Digital distribution and mobile games have made entering the field a lot easier. New funding possibilities such as crowdsourcing through Kickstarter (www.kickstarter.com) or Indiegogo (www.indiegogo.com) have made it even more flexible for nearly anyone to start a game project. Game jams also grow larger each year; Global Game Jam (www.globalgamejam.org) for example has grown from 53 sites, 23 countries, 1650 participants and 370 games in 2009 to 804 sites, 108 countries, 42800 participants and 8606 games in 2018 [20]. It is estimated that one out of five games that reach the marketplace make profit [30]. Therefore, this one profitable game should pay for the development of the other four games.

This paper examines usability in the context of games and game development. Usability has become an important competitive edge in software markets long ago [21], [35], [62]. Users and user organizations benefit from better usability through higher user satisfaction and productivity when the most frequent tasks take less time and users make fewer errors. The development company, then again, benefits from better usability through reduction in time and resources needed for development due to reduced need for changes in late phases. Furthermore, the development company can use improved usability as a competitive edge and potentially increase sales. Better usability can also reduce the number of contacts made to customer support and reduce support costs [4], [14], [35], [44]. As can be seen, there are numerous motivations to invest in usability, even if it is also acknowledged that in the context of game development, these motivations may not all be relevant anymore.

Games are under research activity in Human Computer Interaction (HCI) research, among other disciplines. HCI has addressed games and their development in many respects. One research stream has particularly concentrated on usability in games, concerning either the relationship between usability and games or the process of ensuring usability of games. Usability intuitively is not the most relevant characteristic of good games, but it has still been argued as a relevant aspect, although in need of slight redefinition, as traditional usability concepts such as efficiency may not be particularly important in games ([13], [58]). In game play and game development, motivations for investing in usability have still been found (e.g. [56], [58], [59]. 
$\mathrm{HCI}$ research has already provided plenty of processes and methods for ensuring good usability during software development. Although game development shares many features with software development in general, there are also many specific features in games that pose differing requirements for the development. Especially this study is interested in initially supporting the integration of usability into game development, i.e. there is an assumption that usability has not been institutionalized [65] in game development but instead measures are needed for initially experimenting with usability methods in practice. In game development industry with small start-ups, it is assumed that simple, cheap and fast to use methods are particularly welcomed. In such a context, there may not be resources for hiring usability specialists to take care of usability, but instead developers may have to take care of it among other duties. In such a situation, developers need to be supported in initiating usability work and the methods recommended for them need to be usable for them. There already is HCI research on educating and supporting developers in taking responsibility of usability during development [17], [23], [28], [66], [71]. Although developers' effort is not seen as replacing the need of professional usability specialists, it is still considered as a valuable contribution towards increased usability. Along these lines, in this paper the particular interest will be in supporting game developers to initiate usability work in game development with the support of usable usability methods.

Hence, this paper explores the relationship between usability and games and identifies ways for initially introducing usability activities into game development without professional usability specialists. In such a situation, it is recommended to begin with usability evaluations, which can act as a wake-up call [65] and which, overall, are widely used, successful and efficient usability methods [28], [69]. Of usability evaluation methods, usability inspection methods, such as heuristic evaluation, are less resource intensive and expensive to adopt; hence, they can be relatively easily and quickly adopted in development [28], [38], [50], which is very valuable in game development industry with small start-ups. Additional value of heuristics is that they can also easily be used as guidelines or rules of thumb for design and development. Like Hodent [27] says: “...the earlier UX issues are identified, the less it costs to fix them." To introduce usability into game development, some usability methods have already been introduced, including usability inspection methods [13], [19], [34], [55], [52], [64]. There, however, is a research gap relating to the appropriateness of the developed usability methods in game development, particularly relating to suitability of the methods for game developer use. Some recent studies have alarmingly indicated that heuristic evaluation is not a common usability evaluation method in the game development industry [61] and that game developers rather develop their own usability heuristic lists for each of their games than use existing lists, which they consider too difficult to use [60]. Hence, there is a need to better understand developer experience in game usability - $\mathrm{HCI}$ and games related research should examine 
how usable the existing usability methods are for the game developer as well as develop more usable methods. This paper contributes by taking initial steps along this path.

This paper is structured as follows. The next section presents related HCI literature on usability and its development. The third section discusses game development describing the core concepts, processes and methods. The fourth section reviews research specifically on games and usability. The fifth section presents the research method utilized in this study as well as proposes a set of usable usability heuristics for games, organized through the analytical lenses of game development process phases. The sixth section describes a small-scale survey study conducted on game usability, heuristic evaluation and usability of usability heuristics in game development. The final section summarizes the results of the paper, discusses their implications for theory and practice, presents the limitations of the research and identifies paths for future research.

\section{$2 \quad$ Defining and Designing Usability}

Usability is a central concept in the field of HCI. The most common definitions are certain ISO standard definitions as well as Nielsen's definition. Usability is identified as one of the main software product and system quality attributes in the international standard ISO 9126. It refers to the capability of the product to be understood, learned, and used by users, as well as to appeal to users when used under specified conditions [32]. Standard ISO 9241-11 provides another common definition for usability: "The extent to which a product can be used by specified users to achieve specified goals with effectiveness, efficiency and satisfaction in a specified context of use" [33]. Nielsen, furthermore, defines usability to consist of learnability, efficiency, memorability, errors and satisfaction - the system should be easy to learn to use, the use should be efficient, it should be easy to remember how to use the system, users should not make errors or they should easily recover from them, and the system use should be satisfactory [50].

Usability can be designed and improved through different kinds of usability engineering (UE) and user-centered design (UCD) methods (see e.g. [11], [42], [43], [50], [63]). For example, usability engineering (e.g. [50], [42], [43], scenario-based design (e.g., [63]) and goal-based interaction design (e.g. [11]) have been developed for ensuring usability (and sometimes also usefulness) of the system under development. These methods all contain general phases first relating to understanding users, their needs and tasks and the context of use, to redesigning users' tasks, to creating various kinds of design solutions and to evaluating the design solutions in an iterative manner.

Thus, evaluation of design solutions is an essential step in UE and UCD. It should be carried out at all stages in the system life cycle. Evaluations should be started as early as possible to iteratively improve the design and to generate new ideas. Early evaluations enable fast and cheap improvement of the design. Later, evaluations 
should be carried out to validate that the requirements have been met and to identify requirements for the next version. Usability evaluation can be either empirical, meaning that actual or representative users take part in the evaluations, or they can be inspections that do not involve users, but usability specialists carry out the evaluations relying on some sorts of guidelines, heuristics or standards [33], [38], [50]. Empirical usability testing is the most widely used, the most successful, and the most efficient method for improving usability, while also usability inspection methods are widely utilized [28], [65]. Usability inspections are less resource intensive than empirical usability testing; consequently, they can be more easily and quickly adopted in development ([28], [38], [50]), also by developers ([28], [38]).

The most common usability inspection method is heuristic evaluation, in which evaluators check whether the system conforms to the given usability heuristics. It is relatively easy to learn to use as well as a quick and cost-beneficial method ([38], [50]). Heuristic evaluation is usually performed by a small group of usability specialists by comparing the system against a certain set of good usability principles or rules of thumb called heuristics. Heuristic evaluation involves usability specialists first individually going through the user interface several times, inspecting the general flow and then more specific elements, comparing those with the heuristics. Afterwards, they combine their findings and estimate the severity of the problems. Heuristic evaluation results in the list of usability problems that are connected with the usability principles that they violate. Also fixes to the usability problems should be identified [50]. One of the most recognized and used set of heuristics is the ten heuristics by Nielsen [50], though more precise heuristics have been tailored to serve different areas (e.g. user experience heuristics) and products (e.g. mobile device [29], web development [37] and game usability heuristics [36], [52], [56]).

\section{Defining and Designing Games}

Games are natural to humans, driven by our desire to play and made possible by our ability to pretend [1]. Game is "a type of play activity, conducted in the context of a pretended reality, in which the participant(s) try to achieve at least one arbitrary, nontrivial goal by acting in accordance with rules" [1] or "an interactive structure of endogenous meaning that requires players to struggle toward a goal" [10]. Video games are an art form that combines traditional artistic endeavors such as the visual arts, storytelling and music, with more modern technical and engineering achievements [54]. Games is an interactive, goal-oriented and rule-based activity, where players can interfere and interact with each other [12]. Game must have the following characteristics: light-hearted character, circumscribed in time and place, outcome is unforeseeable, non-productiveness, rule-based and awareness of a different reality [7]. Game has three features: 1) Players who are willing to participate in the game (e.g. for enjoyment, diversion or amusement); 2) Rules which define the limits of the game, and; 3) Goals which give arise to conflicts and rivalry among the players [67]. We see game as a voluntary interactive activity for diversion or amusement, with 
rules, goals and opposition. Rules limit the ways of interaction, goals resolve the winner and opposition either tries to reach the goals first or to prevent the other(s) from reaching them.

Game development process is not that much different from a conventional software development process. There are several process models on how to develop games. The game development process, however, can be divided into three main stages that are pre-production, production and post-production [71] Testing phase can be seen as an independent phase of development that is conducted at the same time with the production phase [8]. Moreover, concept development phase can be seen as another independent stage of the game development process [2], [64]. Therefore, the basic game development process can be divided into following phases (derived from [3], [51]):

Concept: During concept development the main idea of the game is decided and described so clearly that anyone can understand instantly what the game is about [2]. This phase involves high-level design and documentation. Concept development is done after the definition of the initial concept as an iterative cycle consisting of design, development and testing [40]. During this phase, the design team is still small, and errors are easy to fix.

Pre-Production: This is possibly the most important phase, during which every aspect of the game is researched, designed, documented and possibly even developed. Pre-production phase is critical to defining what the game is, how long it will take to make it, how many people are needed and how much it will cost to make it [8].

Prototype: The goal is to create a prototype that shows the basic ideas behind the game and what separates it from other similar games. The prototype can be lo-tech, such as a paper or a miniature version, or a more advanced digital demonstration. The built prototype can be used to test the non-functional requirements of the game that are otherwise difficult to evaluate, such as gameplay. This practice can also be referred to as rapid iterative prototyping [2]. The pre-production stage finishes when the developer has provided a "proof of concept" in the form of a prototype [2].

Production: During the actual production of the formerly designed elements, some changes may occur, but those should be kept minimal. This phase is when the most action happens, most people work at the same time and most time is consumed. The production phase can be divided into three release sub-phases: Alpha, Beta and Gold releases [70].

Alpha: The alpha phase has been defined as the point in the production where in the release the game is mostly playable from start to finish [2] and where a particular build of the game is the first playable version [70]. This is where the full arch of the game is playable, even though there may be bugs or the audio-visual elements are not finished. There is a draft version of the manual and every feature is implemented. At this point, no new features are designed or created. Outside help can be introduced to playtest. In the alpha phase of production, the testing efforts focus on reviewing the features of the game separately and only small testing team is required [2]. 
Beta: When the alpha version of the game has been finalized, the focus of the development shifts from development to finishing the game. In this phase, the goal is to have the game stabilized and remove most of the errors and problems [2]. Therefore, instead of testing the story arch, features and mechanics, beta-tests are all about finding and fixing bugs and fine-tuning the performance on targeted platform(s) and hardware. As the production is nearing its end, the full testing team is brought in [8]. During this phase, everything in the game is finalized or very close to final. Some argue that beta release is the ending point for the production phase, but others consider the production phase to end when the final version - often called gold phase or release phase - has been delivered.

Gold: Gold or release phase can be seen as the climax of the process where the game is ready and waiting to be delivered for the distributors. Development and coding may have stopped for a while, but work has not. There are still distribution channels to work with (playable demos to deliver, trailers to publish etc.) and setting up the support environments.

Post-Release: This phase involves patching the bugs and issues that were not found or fixed before the initial release, sorting the public relations and caressing the community, without forgetting the upgrades, additional contents and special holiday hats. These concluding actions may be considered as of only cursory interest or they may not be done at all as the development team moves on to the next project [8].

These phases are not strict but can and will overlap and some activities such as quality assurance run through the process. Moreover, among authors there are divergent understandings of the phases involved. For example, some authors [2], [70] do not mention the post-release phase or its activities in their game development process descriptions at all and some [3] include more phases than those selected here.

\section{$4 \quad$ Usability and Games}

Game User Research has been around for a few years now. Usability and/or playability in games as well as techniques such as game analytics have gained a lot of attention recently [13], [15], [16], [48]. Increasing number of players and the variety of players have pushed the developers from developing for themselves to developing for everyone. The average Joe or Jane wants to be able to play the game right after installation and wants to be adequately good from the start, without reading any manuals. Thus, usability may provide a competitive edge [31] also in game development e.g. through increased willingness of players to buy a game that has good usability [57], [58]. Usability may contribute to higher user satisfaction and productivity, even though in a slightly different meaning compared to applications supporting work tasks, as game playing is voluntary [63]. Game usability and the quality of the user interface are still very important for players [64]. As more and more game development companies compete for the attention of players, it can be argued that in game development good usability is becoming less an advantage and more a necessity. It 
is easy for players to change to different game if they are not satisfied with the usability of a game, and trial versions, professional game reviews and online gamer communities ensure that a game with bad usability gets a bad reputation fast [46], [58].

What is usability in games then? Well, pretty much the same as in any other software, with some differences [34]. Games are not intended to be productive, - yes, there are some productive games too - but games are primarily supposed to entertain [1]. Usability in games, therefore, addresses issues that hamper users' ability to get entertainment and fun out of the game, areas such as controls, heads-up display and menu structures. There are various good examples and unfortunately quite a few examples of things done wrong (the original Final Fantasy XIV and Fable 3 user interfaces and menu systems, to name two, are some of the most criticized single aspects in a game ever, addressed for example by Morton [46]). With the current reformation of games to a wider audience, usability plays even a stronger role, as the variety of players' gaming skills increases, the games have to adjust to this with tutorials and simplicity (in how you learn to play the game, not in the game). Playability is an extension of usability in games [48]. It adds layers specific to games on top of usability - immersion, character development and how well the game in general comes together for example, whereas playability is a lot more than these [64].

To introduce usability activities into game development, some methods have already been proposed [13], [18], [36], [45], [52], [55], [64]. Fernandez and colleagues [19], Mylly [47] and Sánchez and colleagues [64], among others, have discussed the introduction of usability or playability factors to game development and shown the need for playability elements during development. There are different approaches to bringing usability or playability into development, from player-centered design models such as in Charles and colleagues [9] to heuristic evaluation such as in Desurwire and Wiberg [13]. One of the simplest ways to enhance usability is to introduce heuristics that can be used by every team member, without the need of usability professionals [28], [38], [50], [66]: they provide a cheap and flexible tool for finding issues early and to better understand usability and/or playability [53] that can be used by novice evaluators with good results [22]. Usability heuristics for games were first introduced by Malone [39] in 1980. After quite a few years they have become current again. Today, various sets of heuristics have been introduced to address game usability. Researchers such as Federoff [18], Pinelle and colleagues [56], Brown [6], and Desurvire and Wiberg [13] have produced their own sets and some sets have also been revised later. These sets tend to have dozens of heuristics that very easily appear as confusing and difficult to apply for a game developer who is not experienced in usability. Therefore, the problem with the existing models is that they are targeted at usability researchers and professionals rather than for people without former studies in usability. For these non-usability experts, the heuristics are hard to understand and use.

So far, most of the research has been on establishing a base for the research itself, but the time for putting all this to use in practice has clearly came. Brown [6] has 
taken a more simplified, usability-oriented approach to develop his set of heuristics but we feel that to actually increase usability as well as to help game developers, the heuristics need to support all; design, development and usability; hence too simple a solution does not do it either. The closest approach to ours is that of Desurvire and Wiberg [13], i.e. their PLAY -method, yet the heuristics proposed in this method are again more suitable for usability-oriented people, not for a developer uneducated in usability. In chapter 5, we will propose some examples of usability heuristics that are devised to suit the game developer, in a manner usable to them, divided into different game development phases.

\section{Proposal for Usable Usability Heuristics for Game Developers}

The research process of this paper utilizes both conceptual-analytical and constructive research approaches [41]. The literature review relies on the former, while this research also includes development of developer-oriented game heuristics to be used as a practical tool in game development, which relies on the latter, i.e. design science research (DSR). DSR aims to develop new or improved ways to achieve human goals [24], [41] and it consists of two basic activities: building and evaluating. Building is a process for constructing an artefact for a specified purpose, and evaluating is a process of determining how well the constructed artefact performs in that specified purpose [41].

This paper has the goal of building a set of usability heuristics to fit into the game development context and process, especially for developer use. March and Smith [41] differentiate two cases concerning whether the construct already exists in some form or whether the construct has not existed before in any shape or form. In case the construct is totally new, the contribution of the research comes from the novelty of the artefact and the persuasiveness of the claims that it is effective [41]. In case the construct has already existed in some form - as it is the case in this paper, where both heuristic evaluation and game development process exist in their own separate forms - the contribution of the research lies in the construct being, in some sense, better than the old one [41]. This paper contributes by fitting the heuristic evaluation to the phases of game development process and context to suit the developer use.

The following chapters introduce examples of heuristics that could be introduced and used by everyone involved in game development as guidelines, not just as tools for inspection. The heuristics are kept simple for the best efficiency and divided by the development phase to better integrate them into the game development process. They are based on game usability studies (such as [13], [56], [69]) as well as on game development literature (such as [1], [51], [54], [57]) and on real-life game development experiences in multiple projects and discussions with developers of varying roles and backgrounds. The example heuristics are simple and elemental ones: they were chosen due to their general nature (suitable for most games) and as they are 
easily explainable to everyone, without extensive knowledge in either usability or game development. The proposed usability heuristics were kept simple for the best efficiency while covering the critical areas of game usability in each of the game development phases. Relating to each phase, the selected heuristics try to convey aspects important for ensuring usability of the games as well as aspects particular to the development of games, not software in general.

\subsection{Concept}

The game has (several) clear and visible goals. Players play for a reason, and they must know what they can do and have to do to win the game: e.g. the players explore the game world to find the evil zombie king that they must kill to save the world. Both long- and short-term goals should be visible, long term goals before reaching them and short-term goals straight from their introduction.

The player has a clear role that she can absorb. The player needs to know who she is and how she is ranked in the game context so that she can get a better understanding of the overlying situation and her place in the game world. Without having a clear role, the player can remain disconnected from the game and not feel engaged to it.

The game has a clear audience. Because people are different, not everyone will enjoy the game. One needs to have a clear idea about the audience so that one knows who to develop for. "Everyone" can be an audience, but that does not help the development team, or the actual audience it will have. Designers should know their future players, in order to design and test the concept ideas with appropriate users and to develop the game for them.

\subsection{Pre-Production}

The game is consistent thoroughly. For the game to be believable, the elements in the game need to be consistent. Jumbo-jets in Stone Age are hardly believable but using a pterodactyl as an airplane could work.

Effects and actions of artificial intelligence (AI) are fair, visible and consistent. The AI should not have unfair advantages against the player (i.e. gain resources twice as fast as the player), unless the player chooses so. The actions of the AI should be as visible to the player as her own actions (i.e. troops moving or constructions building). Creating artificial difficulty to the game by "cheating AI" risks alienating the players.

Every platform is thought of independently. Platforms are different, sometimes a lot different (mobile phone vs. personal computer for example) in many ways - from hardware to controllers etc. - therefore the game must be adjusted accordingly to all platforms it is developed for. New technologies and the adaptation levels of these technologies offer possibilities to create something on the side of the real game on other devices (like commanders in Battlefield 3). For 
example, the original version of Final Fantasy XIV had the PC user interface designed to follow the same conventions and limitations as the forthcoming PS3 version, resulting poor usability on PC, which in turn resulted the original PC version failing in the market.

Controls can be customized and are designed for each platform separately. Players are different: for example, some are right-handed, some lefthanded and some may have only one hand or other limitations. The player should be able to adjust the controls so that she can play the game at best possible way. Different platforms have different controllers and some platforms have a multitude of controller options, which should all be designed for independently (within reason). Some platforms and genres have de-facto standards for controls (e.g. WASD), and the designers should follow these when possible for default controls, while giving the players possibility to customize the controls to their liking.

The game is paced to offer challenge and rest. Players are human, the vast majority of humans cannot undergo constant pressure for too long and too long easy periods can get boring. Keep the player engaged in the game by keeping the tension close but allow her to stop to think and breathe every now and then.

\subsection{Prototype}

The hook should be obvious. The prototype needs to prove that the game is worth making, selling and most importantly playing. Do not just reinvent the wheel, improve it. Provide the hook of the game in the prototype.

The theme and genre are obvious. The prototype shows what the game is about and in what setting, the level of reality (arcade versus simulator) and the main genre (sports, action, role play etc.). The players set their expectations and use mental models based on their existing experience on themes and genres. If the theme or genre is not obvious, players do not know how to approach the game and may be off put from the start.

The rules and opposition are obvious. The rules define how the game is played and opposition is the challenger for the player. These can be demonstrated at general level at this point. The rules and opposition should be clear, fair, and give the player possibility to learn, develop and progress.

The over-arching goal is obvious. The Player is aware of what the final goal of the game is, even if not yet able to complete that goal.

\subsection{Production}

The player should be able leave or quit the game at any point. Players have real lives, therefore they should be able to leave (pause) or quit (save) the game at any point without losing too much of their progress. This may not be applicable in competitive multiplayer games, but a quit or pause should be included in every single 
player game at least and should be taken into account through whole production phase. The player should not have to abandon a lot of progress in the game because of a sudden real-life need.

Produce for the player. Adjusting to tight schedules often may lead to cutting corners and reducing features, which is understandable, but these cuts should not affect the general game play. Through whole production phase, leave "cool to have" features out if that means you can perfect the necessary features, save time on places that are optional to finish the mandatory and often visited places.

\subsection{Alpha}

The player knows what to do and how to do it. The game clearly shows what the player needs to do - a first goal - and the means to do it, so that the player can start playing meaningfully right from the beginning. The original version of Final Fantasy XIV gained notoriety amongst players for leaving them wander in the game world not knowing what to do, how to do it and why to do it.

The player does not repeatedly make the same mistakes. Repetition can frustrate the player easily and repeated mistakes frustrate even faster. Alpha is the first phase where you can really spot these design flaws through player testing, as the developers play tests are biased (developers know how it should be done).

Basic controls are easy to learn, but expendable for more experienced players. The player learns the basic controls fast and can play the game right from the beginning. Advanced players may require more advanced controls to achieve things faster or with fewer actions (e.g. quick keys for production or building).

Menus are simple, self-explained and intuitive. Menus are part of the game and the overall experience too. The menus should be designed and structured to support the game play. Disguising different options as part of an image for example might seem like a good idea, but if it makes it slower or harder for the player to start the game, it does not support the game play and can potentially drive players away from the game.

\subsection{Beta}

The game provides accurate, timely and adequate feedback. Players need to know and realize they are progressing towards the goal(s) of the game. Giving them feedback of their progress will encourage them to continue playing (progressive feedback) and feedback about their current situation and future will make it a lot clearer how to proceed onward (informative feedback). Most of the core should at this point work like intended, but it is not too late to increase the amount of information the player receives.

The challenges are worth completing and give a positive feeling. The challenges can be very hard, but if the players are given a feeling of success, they will carry on trying. Having sub-goals in a longer challenge (pacing) to provide constant 
stream of minor successes can help in reducing player stress. The reward(s) for completing a challenge should be on par with the challenge level, greater challenges should provide greater rewards.

User interface has enough information but does not interfere with game play. Different games have different requirements for the user interface. Health indicators, ammunition information, mini-maps, compasses, skill bars and every other thing there may be are supposed to support the player and the game play, not to obstruct her view or ability to play.

Similar forms should have similar actions. A sign for example should always be a sign, not sometimes a mere sign and sometimes a button as well to avoid confusion and frustration. Less learning is more playing.

The game and the different elements in the game are balanced. In the beta phase of the production it is time to make sure that the different elements in the game are balanced so that the players' skills and abilities define the game outcome, more than (bad) early game decisions or imbalanced mechanics.

\subsection{Gold}

The game is ready to play out of the box. The player does not need to install any updates or patches prior to game play. Notify the player that there are updates available, but do not force her to install unless the updates are critical for the game (i.e. online competitive game, where everyone needs to have the same version). The player experience suffers considerably, if the player starts the new game with high expectations, only to have to wait for a massive update package to be downloaded and installed.

There is an adequate manual shipped with the game. The manual included in the release (preferably in physical form, electronic manuals are hardly ever even opened) has enough information so that the player can quickly look up basic instructions and well-known issues. The player should not have to search for websites, discussion forums or community sites for information.

All required third-party software is included in the release. If the game requires any third-party software (DirectX, PunkBuster etc.), they are all included in the release to make it as easy as possible for the player to start the game. It may be plausible to add links or web-installers for the requirements these days, as internet is widely available and software updates common.

The community building is supported in several ways. Playing games is a social activity and a flourishing community is an important part of a successful game experience. Building and caring the community properly also creates ground for your next release or other games, as word spreads quickly. 


\section{$5.8 \quad$ Post-Release}

There is an up-to-date FAQ about known issues and their (possible) fixes. The players can find information for problem situations in one, centralized place, so that they can overcome and/or fix issues as easy as possible.

There is a change log available for every update and patch. Provide information about changes to game mechanics and content etc. so that the players can adjust their game play accordingly (if needed) and are well informed about fixed bugs and errors.

New content is consistent with the original game. The new content should support the existence of the original game and the original game experience, which can be extended and expanded, but not overwritten.

\section{Feedback from The Field}

A small survey was conducted in February 2020 among Finnish game developers of wide variety among company sizes and roles within the companies on game usability, on the role of heuristic evaluation in the companies and on the usability of the usability heuristics presented in the literature as well as of those proposed in this paper. There were 8 respondents in the survey.

When asked about what usability means to the respondents, one answer is quite close to what we think of (game) usability: "Usability is one of the design goals that should be pursued during development to make the product as accessible, intuitive and transparent as necessary to its core audience.". This gives motivation that usability activities should be incorporated to the development cycle as a whole. Another answer underlines giving space to the players to learn the game and the ability to customize: "Usability in a game is how well a player can use and engage with the game itself. Poor usability is normally bad UX design and no customization for the player to mold the game around how they want to play (such as moving controls to fit left and right handedness), along with no space for the player to learn how the game works.".

As for the results, $75 \%$ of the respondents found usability in games to be of high importance (4.6 average out of 5) and majority of companies the respondents worked with valued usability very important in their products (4.1 out of 5). However, $50 \%$ of the respondents did not currently use heuristic evaluation as a usability tool, yet $57 \%$ would like to use some form of heuristics to support their work and quoting one answer straight from open feedback question rather nicely sums up the general feeling among the respondents: "UX is very important. Every team / game / company should have one person full time on this.". When asked why they were not using heuristics in their projects there was answers such as: "Normally it's based on time constraints. Spending time to design and create a fully heuristic sequence or space for players to learn the game themselves is a very difficult and time consuming task, that also doesn't translate to other projects quite as well as something more structured 
and conventional. However, that doesn't mean that I would like to have the time to create such a space for players so that they can learn the way the game works in their own way.", which indicates that incorporating heuristics as a development tool as well could be potentially both saving time and money.

The respondents were asked about usability of a variety of different heuristics randomly chosen from Federoff [18], Nielsen [50], Desurvire and Wiberg [13] and usability heuristics proposed in this paper. The respondents were not told from where the heuristics are from, or by whom and no further descriptions were given. The survey inquired how easy to apply would a given heuristic be in a current project(s).

Two heuristics by Federoff [18] gained the highest average points and our three heuristics together with Nielsen's heuristics followed very close behind, leaving the impression that simpler and/or less error-prone heuristics are easier to apply. Open question about what kind of heuristics would help in the respondents area of expertise asked for "Live Ops and service heuristics" along with "More understanding", which we see as need for a wider set of heuristics that includes the whole range of game development from concept to live operations and serving the user base, but also a set that explains what and why the heuristics are. The heuristics proposed in this paper are answering those questions and work as a basis for a more detailed and wider spectrum of coverage across game development as a whole.

\section{Concluding Discussion}

This paper addressed the topic of usability in game development. Game development industry, although growing fast and already including large and successful companies, consists also of a huge number of small-to-medium-sized enterprises and startups. For these companies, practical, usable, developer-oriented tools for ensuring game usability are needed, as these companies do not have resources for hiring usability specialists. This paper reviewed the concept of game usability and existing research on usability methods for game development as well as proposed a set of usability heuristics as a usable, developer-oriented tool during the game development process.

In case the construct has already existed in some form - as it is the case in this paper, where both heuristic evaluation and game development process exist in their own separate forms - the contribution of the research lies in the new form of the construct being, in some sense, better than the old one [41]. We propose that the usability heuristics fitting the game development process and context, as presented in this paper, are more usable [32], [33], [46] than the existing game usability heuristics, which are not being favored by the majority of the game companies [60]. The usability of the heuristics is improved especially in the sense of efficiency and effectiveness [33] that hopefully also lead to improved developer satisfaction [33]. The literature indicates that the game companies view the existing game usability heuristics as being too difficult to use, too expensive, requiring too much resources, un- 
known to them, and unsuited for their development practice [60]. The proposed usability heuristics were kept simple for efficiency while covering the critical areas of game usability in each of the game development phases to ensure effectiveness. Dividing the game usability heuristics into the different development phases aims to help to integrate them into game development and allows the game developers to concentrate on a smaller number of important game usability heuristics in each phase. We argue that this new approach to game usability heuristics makes it easier for the game developers to use these heuristics in their game development process and context and thus to improve the usability of the games they develop.

A small-scale survey study on game usability, heuristic evaluation in game development and usability of the usability heuristics proposed in the literature as well as in this study was carried out. The results show that the respondents and companies value usability of games high, but they do not necessarily use heuristic evaluation for improving usability in the development. Then again, they indicate interest in using heuristic evaluation in the future. In the survey, simpler and less error-prone heuristics were found easiest to apply, such as we propose in this paper. Question about the need for heuristics in the respondent's area of expertise showed the need for a wider spectrum of heuristics than what can be found in current literature, together with the need to know more about the heuristics (what, why, when to use).

The study has both theoretical and practical implications. First of all, through reviewing the concept of game usability and existing research on usability methods for game development as well as through characterizing the existing game development industry - i.e. there being a huge number of small enterprises and start-ups without much resources to invest in usability - the paper revealed the need for a practical, usable and developer-oriented tool to suit the game development process that has been lacking in the existing research [59], [60], [61]. Second, the proposed and evaluated usability heuristics for games aim at meeting this need. Other researchers as well as game developers can benefit from the contribution of this paper by experimenting with the heuristics during game development. Researchers can extend this work by testing the fit of particular heuristics in the respective game development phase as well as by refining or developing new game usability heuristics to fit a particular game development phase.

As a limitation of this paper, the explored usability heuristics for game development have not been comprehensively empirically tested to verify their fit, usefulness and ease of use. We also acknowledge that heuristic evaluation conducted by novice evaluators (i.e. developers) is not a perfect solution for improving game usability, but it is better than nothing (see also [17], [23], [71]). This paper focuses on usability evaluation and practical developer-oriented usability tools, ignoring issues such as usability requirements, usability design and the institutionalization of usability [65] in game development, even if they definitely are significant to consider in game development.

There is still a need for more research on introducing usability activities into the game development context and process. More research also needs to be done related 
to introducing game usability heuristics into game development. The proposed usability heuristics should be more comprehensively empirically tested in game development to verify the fit and usefulness of these heuristics in their particular game development phase. Moreover, heuristic evaluation is just one particular usability method and usability evaluation is just one particular usability activity. Empirical usability testing, usability design and usability requirements should also be considered in game development (see also [45], [61]). Altogether, more research needs to be done related to institutionalizing usability activities [65] in game development. Finally, the potential costs and benefits of usability activities in game development context could be researched further [59].

\section{References}

1. Adams, E.: Fundamentals of Game Design, 2nd ed. New Riders, Berkeley. (2010)

2. Bates, B.: Game Design, Second Edition. Boston, MA, USA: Course Tech. (2004)

3. Bernhaupt, R.: Game User Experience Evaluation. pp. 4-5. Springer, Switz. (2015)

4. Bevan, N.: Cost Benefit Analysis version 1.1 Trial Usability Maturity.Serco. (2000)

5. Blow, J.: Game development: Harder than you think. ACM, 1(10), pp. 28-37. (2004)

6. Brown, M.: Evaluating Computer Game Usability: Developing Heuristics Based on User Experience. The 2nd Annual Human Computer Interaction Conference. (2008)

7. Callois R.: Les jeux et les homes. Gallimard, Paris, France. (1957)

8. Chandler, H.: The Game Production Handbook, 2nd Ed. J. \& Bartlett, USA. (2008)

9. Charles, D., McNeill, M., McAlister, M. Black, M., Moore, A., Stringer, K., Kücklich, J., Kerr, A.: Changing Views - Worlds in Play. In Proc. DiGRA. (2005)

10. Costikyan, G.: I Have No Words \& I Must Design: Toward a Critical Vocabulary for Games. Proc. of Computer Games and Digital Cultures Conference. Tampere, Finland. (2002)

11. Cooper, A., Reimann, R.: About face 2.0: the essentials of interaction design. Wiley, Indianapolis. (2003)

12. Crawford, C.: Chris Crawford on Game Design. New Riders. (2003)

13. Desurvire, H., Wiberg, C.: Game Usability Heuristics (PLAY) For Evaluating and Designing Better Games: The Next Iteration. (2009)

14. Ehrlich, K. and Rohn, J.: Cost Justification of Usability Engineering. In: Bias, R., Mayhew, D. (eds.): Cost- Justifying Usability. Acad. Press, pp. 73-110. (1994)

15. El-Nasr, M.S, Desurvire, H., Nacke, L., Drachen, A., Calvi, L., Isbister, K., Bernhaupt, R.: Game User Research. In Proc. of CHI EA, NY, pp. 2679-2682. (2012). 
16. El-Nasr, M.S., Drachen, A., Canossa, A.: Game Analytics. Maximizing the Value of Player Data. Springer-Verlag, London. (2013)

17. Eriksson, E., Cajander, A., Gulliksen, J.: Hello World! - Experiencing Usability Methods without Usability Expertise. INTERACT 2009, pp. 550- 565. (2009).

18. Federoff, M.: Heuristics and Usability guidelines for the creation and evaluation of fun in video games, thesis, University of Indiana. (2002)

19. Fernandez, A., Insfran, E., Abrahão, S., Carsí, J. Á., Montero, E.: Integrating Usability Evaluation into Model-Driven Video Game Development. In: M. Winckler, P. Forbrig, R. Bernhaupt, Human-Centered Software Engineering. Springer Berlin. pp. 307-314. (2012).

20. Global Game Jam (2019), http://globalgamejam.org/history. Access. June 27, 2019

21. Grudin, J.: Interactive Systems: Bridging the Gaps between Developers and Users. IEEE Computer 24(4), 59-69. (1991)

22. Hearst, M. A., Laskowski, P., Silva, L.: Evaluating information visualization via the interplay of heuristic evaluation and question-based scoring. In Proc. of the 2016 CHI Conference, pp. 5028-5033. (2016).

23. Hertzum, M., Jacobsen, N. E.: The evaluator effect: A chilling fact about usability evaluation methods. International J. of HCI, 13(4), 421-443 (2001)

24. Hevner, A.R., March, S.T., Park, J.: (2004). Design research in information systems research, MIS Quarterly, 28:1, 75-105.

25. Hiltunen, K., Latva, S., Kaleva, J-P.: Peliteollisuus - kehityspolku, Tekes. (2013)

26. Hiltunen, K., Latva, S., Kaleva, J-P.: Finnish Game Industry Report, Neog. (2017)

27. Hodent, C.: The Gamer's Brain: How Neuroscience and UX Can Impact Video Game Design. CRC Press, Boca Raton. Chapter 16. (2018)

28. Holzinger, A.: Usability engineering methods for software developers. Commun. ACM 48(1), 71-74 (2005)

29. Inostroza, R. Rusu, C., Roncagliolo, S., Jimenez, C., Rusu, V.: Usability Heuristics for Touchscreen-based Mobile Devices. Proc. of ITNG 2012, pp. 662-667. (2012).

30. Irwin, M. J.: Cooking Up A Blockbuster Game. Forbes (2008)

31. Isbister, K., Schaffer, N.: Game Usability: Advice from the Experts for Advancing the Player Experience. CRC Press, Boca Raton. (2008)

32. ISO 9126: International standard. (2001)

33. ISO 9241-11: International standard. (1998)

34. Jørgensen, A. H.: Marrying HCI/Usability and computer games: a preliminary look. In Proceedings of the third Nordic conference on Human- computer interaction. ACM, New York, pp. 393-396. (2004)

35. Karat, C-M.: A Business Case Approach to Usability Cost Justification. In Bias, R., Mayhew, D. (eds.): Cost-Justifying Usability. Academic Press, pp. 45-70. (1994) 
36. Korhonen, H., Koivisto, E.M.I.: Playability heuristics for mobile multi-player games. In Proceedings of the 2nd international conference on Digital interactive media in entertainment and arts, pp. 28-35. ACM. (2007)

37. Levi, M. D., Conrad, F. G.: A heuristic evaluation of a World Wide Web prototype. Interactions, 3(4), 50-61 (1996)

38. Mack, R., Nielsen, J.: Executive summary. In Usability Inspection Methods, Nielsen, J., R. Mack (eds.). John Wiley \& Sons, Hoboken, NJ, pp. 1-23. (1994)

39. Malone, T. W.: What makes things fun to learn? heuristics for designing instructional computer games. SIGSMALL '80 Proceedings of the 3rd ACM SIGSMALL symposium and the first SIGPC symposium on Small systems. ACM. New York. Pp. 162-169. (1980)

40. Manninen, T.: Pelisuunnittelijan käsikirja - Ideasta eteenpäin. Rajalla, Oulu, (2007)

41. March, ST., Smith, G.F.: Design and natural science research on information technology. Decision support systems, 15(4), 251-266 (1995)

42. Mayhew, D.: Strategic Development of Usability Engineering Function. Interactions, 6(5), 27-34 (1999)

43. Mayhew, D.: The usability engineering lifecycle: a practitioner's handbook for user interface design. Morgan Kaufmann Publishers, San Francisco. (1999)

44. Mayhew, D., Mantei, M.: A Basic Framework for Cost- Justifying Usability Engineering. In Bias, R., Mayhew, D. (eds.): Cost-Justifying Usability. Acad. Press, pp. 9-43. (1994)

45. Medlock, M. C., Wixon, D., Terrano, M., Romero, R., Fulton, B.: Using the RITE method to improve products: A definition and a case study. U. Prof. As., 51. (2002)

46. Morton, P.: Learning from Fable III's UX Mistakes, UX booth, http://www.uxbooth.com/articles/learning-from- fable-iiis-ux-mistakes/ (2011)

47. Mylly, S.: Genre-Based Modular Heuristic Models for Improving Usability During Game Development. Oulu: Oulun yliopisto. (2011)

48. Nacke, L.: From playability to a hierarchical game usability model. In Proc. of Future Play '09, ACM, New York, pp. 11-12. (2009)

49. Newzoo: Global Games Market Report. Q2 2016 Update. (2016)

50. Nielsen, J.: Usability engineering. Academic Press, Boston. (1993)

51. Novak, J.: Game Development Essentials: An Introduction, Delmar, USA. (2012)

52. Paavilainen, J.: Critical review on video game evaluation heuristics: social games perspective. In proc. of the International Academic Conference on the Future of Game Design and Technology, pp. 56-65. (2010)

53. Paavilainen, J., Korhonen, H., Koskinen, E., Alha, K.: Heuristic Evaluation of Playability: Examples from Social Games Research and Free-to-Play Heuristics . In: Games User Research, pp. 257-280. (2018) 
54. Pagulayan, R., Steury, K.: Beyond Usability in Games. Funology, 11(5), ACM, New York, 70-71. (2004).

55. Phan, M. H., Keebler, J. R., Chaparro, B. S.: The development and validation of the game user experience satisfaction scale (GUESS). Human Factors, 58(8), 1217 1247 (2016)

56. Pinelle, D., Wong, N., Stach, T.: Heuristic evaluation for games: usability principles for video game design. In Proc. of CHI '08, New York, pp. 1453-1462. (2008).

57. Rabin, S.: Introduction to Game Development, Second Edition, Course Technology. Boston, USA. (2010)

58. Rajanen, M., Marghescu, D.: The impact of game usability to player attitude. Proc. of 29th Information Systems Research Seminar in Scandinavia, pp. 1-17. (2006)

59. Rajanen, M., Rajanen, D.: Usability benefits in gamification. In Proc. of the 1st GamiFin Conference. Pori, Finland. pp. 87-95. (2017)

60. Rajanen M., Rajanen, D.: Heuristic evaluation in game and gamification development. In Proc. of the 2nd GamiFin Conference. Pori, Finland. (2018)

61. Rajanen, M., Tapani, J.: A Survey of Game Usability Practices in North American Game Companies. In proc. ISD2018, Lund, Sweden. (2018)

62. Rosenbaum, S., Rohn, J., Humburg, J.: A Toolkit for Strategic Usability: Results from workshops, Panels, and Surveys. In Proc. CHI, NY, pp. 337-344. (2000).

63. Rosson, M., Carroll J.: Usability Engineering: Scenario-based Development of Human-Computer Interaction. Morgan-Kaufman, San Francisco. (2002)

64. Sánchez, J. L., Zea, N. P., Gutiérrez, F. L.: From Usability to Playability: Introduction to Player-Centered Video Game Development Process. In: Human Centered Design, HCII 2009 pp. 65-74. (2009)

65. Schaffer, E.: Institutionalization of Usability: A Step-by-Step Guide. Ad.-W. (2004)

66. Seffah, A., Metzker, E.: The obstacles and myths of usability and software engineering. Communications of the ACM 47(12). 71-76 (2004)

67. Smed, J., Hakonen, H.: Towards a Definition of a Computer Game. Technical Report 553, Turku Centre for Computer Science. (2003)

68. Suits, B.: The Grasshopper: Games, Life and Utopia / Bernard Suits; with a new introduction by Thomas Hurka, Broadview Press. (2005)

69. Sykes, J., Federoff, M.: Player-Centered Game Design. Proceeding CHI '06 extended abstracts on Human factors in computing systems, pp. 1731-1734. (2006)

70. Vuorela, V.: Pelintekijän käsikirja. Helsinki, Finland: BTJ Finland Oy. (2007)

71. Øvad, T., Larsen, L. B.: How to reduce the UX bottleneck-train your software developers. Behaviour \& Information Technology, 35(12), 1080-1090 (2016) 